\title{
A Rare Case of Peritoneal Loose Body (Mice) in Left Sided Inguinal Hernial Sac
}

\author{
Deep N Patel*, Rajnish R Patel, Hitendra K Desai and Rajesh K Patel \\ Department of General Surgery, B.J. Medical College \& Civil Hospital, Ahmedabad, Gujarat, India.
}

*Corresponding author: Deep N Patel, Post-Graduate Junior Resident Doctor, Department of General Surgery, B.J. Medical College \& Civil Hospital, Ahmedabad, Gujarat, India.

Received Date: December 14,2020

Published Date: December 23, 2020

\begin{abstract}
Background: Peritoneal loose bodies or peritoneal mice are calcified pieces of necrotic tissue found in the peritoneal cavity. They are usually asymptomatic and small, ranging from 0.5 to $1 \mathrm{~cm}$ in size, but rarely may be up to 5 to $10 \mathrm{~cm}$ in size [1]. Peritoneal loose bodies develop from Appendices epiploicae that has undergone axial rotation followed by necrosis of its pedicle and detachment. Peritoneal loose bodies are incidental finding at laparotomy [2] but they are rarely described in hernial sac in the literature. By this article I have attempted to describe the rare case report of this subject to add to the scarce literature on this subject

Case report: One such loose body of $2 * 2 \mathrm{~cm}$ size was found intraoperatively in a 45 -year-old male patient presented to civil hospital Ahmedabad with left sided inguinal hernia \& operated at Civil Hospital, Ahmedabad in the indirect inguinal hernial sac. Left inguinal hernioplasty was done and excised sac with containing loose body was sent for histopathology which confirmed it to be a peritoneal loose body.
\end{abstract}

Keywords: Loose body; Mice; Appendices epiploicae; Inguinal hernia; Hernioplasty

\section{Introduction}

Peritoneal loose bodies or peritoneal mice are calcified pieces of necrotic tissue found in the peritoneal cavity. They are usually asymptomatic and small, ranging from 0.5 to $2.5 \mathrm{~cm}$ in size, but rarely may be up to 5 to $10 \mathrm{~cm}$ in size [1]. Peritoneal loose bodies almost never cause any symptoms. They are mostly found incidentally during laparotomy [2]. One or more may be found in hernia sac or in the pouch of douglas. The loose body may come from appendix epiploica that has undergone axial rotation followed by necrosis of its pedicle $\&$ detachment via sequential processes of torsion, infarction, saponification, and calcification [3]. They are also found in those who are suffering from subacute attack of pancreatits [4]. These hyaline bodies attain the size of a pea or bean and contain saponified fat surrounded by fibrin [4]. Peritoneal loose bodies almost never cause any symptoms. In most cases, PLBs are asymptomatic and are no larger than a pea; they have little or no clinical significance and do not require any specific treatment. However, they occasionally grow to larger dimensions and cause symptoms such as intestinal obstruction and urinary retention [3]. The occurrence of peritoneal loose body in the indirect inguinal hernia sac is a relatively rare finding. It can mimic malignancy, so It is usually diagnosed intraoperatively [5] \& confirmed by histopathological examination.

\section{Case Presentation}

45-year-old Hindu gentleman laborer residing at Mandsaur, M.P, India. came to civil hospital Ahmedabad surgical opd with c/o left sided groin swelling for 1 year, patient was relatively asymptomatic before 1 year then he developed swelling in left inguinal region which was of insidious onset, first appeared in inguinal region then progressed to the scrotum, gradually increased to present size, increasing in size during straining, exercise and heavy weight lifting \& not reducing after lying down. It was not associated with pain, cough, constipation \& urinary symptoms. Patient was operated for right inguinal hernioplasty 10 years back at private hospital in 
Mandsaur, M.P. There was no past history of tuberculosis/diabetesmellitus/hypertension/jaundice/blood transfusion/any other previous surgeries. Patient was vitally stable.

\section{Inguino-Scrotal Region Examination}

On inspection-Approx. $6 * 4 \mathrm{~cm}$ sized, pyriform shaped swelling was visible extending from left inguinal region upto the base of scrotum, not reducing spontaneously after lying down. Visible expansile cough impulse over the swelling was present. Skin over swelling was normal and penis was in normal position. Left testis was visible separately from the swelling. Another approx, $2 * 2$ $\mathrm{cm}$ sized globular lump with smooth surface and well-defined margins was present over the pyriform swelling confined to left inguinal region which was only visible during coughing and goes back after lying down. On right side, $5 \mathrm{~cm}$ right hernioplasty scar was present and rest of the findings were normal. On palpationInspectory findings were confirmed. Temperature over the swelling was normal and tenderness was absent, Swelling was of doughy consistency and get above the swelling was not possible. Swelling was located above and medial to pubic tubercle. Left testis was separately palpable. After manual reduction of swelling, Deep Ring Occlusion Test was positive (no impulse or hernia bulge medial to occluding finger), Ziemann's Test-impulse was felt over index finger (deep ring). Another approx. $2 * 2 \mathrm{~cm}$ sized lump, firm to hard in consistency, globular shaped, freely mobile lump with smooth surface and well defined margins was present over the pyriform swelling localized to left inguinal region and was palpable only during coughing and freely moves back after lying down. On right side, no cough impulse was felt.

1. On percussion-dull note was present over swelling.

2. On auscultation-no bowel sounds were heard.

3. On Per-Rectal \& Digital Rectal Examination-Prostate was $1+$

4. Malgaigne's bulge was absent.

5. Respiratory System examination was normal.

6. Per Abdomen was soft, nontender.

\section{Investigations}

\section{USG (abdomen+pelvis) with non-contrast-CT cut correlation}

Approx.33 mm sized defect in left inguinal region through which bowel and mesentery have herniated out on coughing reaching upto base of scrotum. Minimal fluid is noted in left scrotal sac.Approx.16*16 mm sized heterogeneously echo textured lesion without significant internal vascularity is noted in left spermatic cord near root of scrotum p/o spermatic cord lipoma or peritoneal mice, Prostate $=20 \mathrm{ml}$ in size \& post voidal residual volume is practically nil.

\section{Intra-operative findings}

Left sided lichte instein's tension free hernioplasty was done and on examination there was indirect hernia sac was present containing mesentery and containing approx. $2 * 2 \mathrm{~cm}$ sized boiled egg like structure embedded within it (Figure 1).

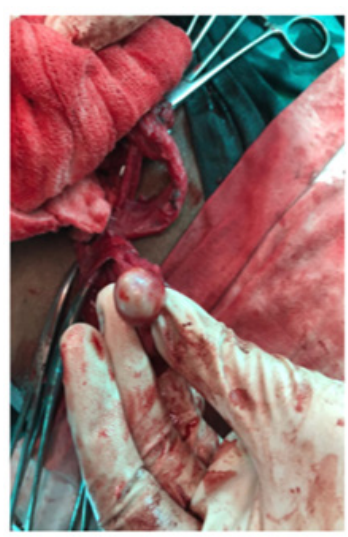

Figure 1: intra-op picture of peritoneal loose body in indirect hernial sac.

Excessive sac with peritoneal boiled egg like structure was excised and sent for histopathological examination (Figure 2).

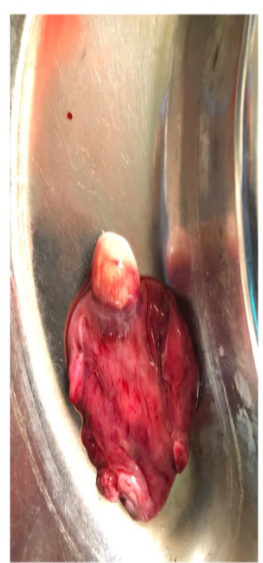

Figure 2: Biopsy specimen containing peritoneal loose body.

\section{Histopathological examination}

Section shows proliferation of fibrofatty tissue with stromal proliferation and calcification s/o Peritoneal Loose Body (MICE)

\section{Post-op course}

Patient was discharged on post-op day 2 without any complaints. After regular cleaning \& dressing \& closed follow up, Stitches were removed on post-op day 10. After that patient has uneventful post-op course.

\section{Discussion}

Peritoneal loose bodies or peritoneal mice are calcified pieces of necrotic tissue found in the peritoneal cavity. They are usually asymptomatic and small, ranging from 0.5 to $2.5 \mathrm{~cm}$ in size, but 
rarely may be up to 5 to $10 \mathrm{~cm}$ in size [1]. They are usually incidental findings during laparotomy [5] and confirmed by histopathological examination. It is rare however possible for epiploic appendigitis to result in a peritoneal loose body. Peritoneal loose body is a freefloating mass of dead fibrous tissue surrounded by several layers of calcification. The loose body is the result of torsed, infarcted or detached epiploic appendages that eventually become fibrotic (inflammation and scarring) masses. Thus, it occurs via axial rotation of appendices epiploicae followed by necrosis of its pedicle \& detachment via sequential processes of torsion, infarction, saponification, and calcification [3]. If the loose body becomes large enough it can cause urinary retention or bowel obstructions [5]. These cases are reported in literature. Epiploic appendages are also called appendices epiploicae. The appendages themselves are 50-100 appendages that are oriented in two rows anterior and posterior. The appendages are parallel to the superficial section of the taenia coli. Furthermore, the appendages are between 0.5 and 5 $\mathrm{cm}$ long, each appendage is attached with one or two arterioles and a venule within vascular stalks attached to the colon [6]. Torsion of the appendages can cause ischemia which can cause painful symptoms that mimic other conditions such as diverticulitis, and appendigitis; however, it is rare. The pain associated with the inflamed appendages is located in the left and sometimes in the right lower abdominal quadrant. Diagnosis of epiploic appendigitis can be challenging due to its infrequency. Epiploic appendigitis is the major pathological factor for the development of peritoneal loose bodies [6]. Radiological investigation of choice for peritoneal loose body is CT scan [1]. CT shows centrally calcified mass [1]. For confirmation, ultimate diagnosis \& therapeutic modality of choice for the peritoneal loose body is surgical exploration [5].

\section{Conclusion}

Peritoneal loose bodies are extremely rare in the location like indirect inguinal hernia sac. It can present a challenging diagnostic problem to the surgeon, with confusing findings that can point towards malignancy. It often requires a number of investigations, but even with these investigations, operative exploration, either open or laparoscopic, can be the ultimate diagnostic and therapeutic modality. The mobility of these calcified lesions may give a preoperative clue to the etiology. It is important to distinguish peritoneal loose bodies from neoplastic or metastatic lesions. This case report adds to the scarce literature \& serves to alert the practicing surgeons/urologist to consider a peritoneal loose body in the list of differential diagnosis of incidentally detected calcified mass while surgical exploration.

\section{Acknowledgement}

I am extremely thankful to my parents, my brother \& sister-inlaw and my teachers \& department of general surgery-civil hospital, Ahmedabad for encouraging me \& guiding me for this case report.

\section{Conflict of interest}

No conflict of interest

\section{References}

1. Dr Yair Glick, Dr Hamish Smith (2020) Peritoneal loose body. radiopedia online articles.

2. Ajit Sewkani, Aruna Jain, Maudar Kk, Subodh Varshney (2011) 'Boiled egg' in the peritoneal cavity-a giant peritoneal loose body in a 64-yearold man: a case report. J Med Case Rep. 7(5): 297

3. Harrigan AH (1917) Torsion and Inflammation of the Appendices Epiploicae Ann Surg. 66(4): 467-478.

4. Williams, Norman S, O’Connell, Ronan P, Mc Caskie, AW (1947) Bailey \& Love's short practice of surgery. 27th edition.

5. Munzir Obaid, Salahddin Gehani (2018) Deciding to Remove or Leave a Peritoneal Loose Body: A Case Report and Review of Literature. Am J Case Rep 19: 854-857.

6. Wikipedia.org-online article-peritoneal loose body/appendices epiploicae/epiploic appendigitis 